doi:10.17659/01.2017.0021

Journal of Case Reports 2017;7(1):75-77

\title{
Vasculitic Ulcers in a Young Female with Dermatomyositis: An Unusual Case
}

\author{
Sankha Shubhra Chakrabarti, Annesh Bhattacharjee, Indrajeet Singh Gambhir \\ Department of General Medicine, Institute of Medical Sciences, Banaras Hindu University, Varanasi, Uttar Pradesh, India.
}

\section{Corresponding Author: \\ Dr. I S Gambhir \\ Email: iac2k10@gmail.com}

This is an Open Access article distributed under the terms of the Creative Commons Attribution License (creativecommons.org/ licenses/by/3.0).

Received

Accepted

Published

November 21, 2016

January 23, 2017

February 25, 2017

\begin{abstract}
Background: Vasculitic ulcers are one of the rarer cutaneous manifestations of dermatomyositis. They are considered to be a predictor of underlying malignancy. Case Report: We describe a case of extensive cutaneous vasculitic ulcers in a young female patient of dermatomyositis without a background of systemic connective tissue disorder or malignancy. Therapy with steroids and methotrexate resulted in swift remission in the patient. Conclusion: Cutaneous leukocytoclastic vasculitis in a patient of dermatomyositis shows good response to steroids complemented by steroid sparing agents.
\end{abstract}

Keywords: Connective Tissue Diseases, Dermatomyositis, Methotrexate, Neoplasms, Steroids, Leukocytoclastic Vasculitis.

\section{Introduction}

Dermatomyositis is one of three inflammatory myopathies and is characterized by proximal predominant muscle weakness as well as cutaneous manifestations. It has an incidence between 1.9 to 7.7 cases/million population/year and a female preponderance [1]. The strong association of this entity with malignancies is a notable feature with ovarian, gastrointestinal, breast and lung tumors being the commonest. Dermatomyositis in the absence of malignancy or another connective tissue disorder is a rare entity [2]. The typical cutaneous manifestations of dermatomyositis are the Gottron's papules (violaceous maculopapular eruptions on the knuckles and elbows); the heliotrope rash (purple colored rash over the eyelids, cheek, forehead and bridge of nose); V-sign; Shawl sign and Holster sign. Other nonspecific signs include calcinosis, mechanic's hands, panniculitis and lipodystrophy. Cutaneous leukocytoclastic vasculitis is a less common dermatological manifestation of dermatomyositis. We describe a young female with dermatomyositis who developed extensive vasculitic ulcers of the skin in absence of other connective tissue disease or malignancy.

\section{Case Report}

A 21 year old unmarried female presented with multiple painful and pruritic purple maculopapules, reticular lesions and patches extensively distributed over her back, flexor and extensor surfaces of bilateral upper limbs, left thigh and right axilla. Most of the lesions were ulcerated and covered with slough [Fig.1,2]. In addition, she had gradually progressive symmetrical proximal limb weakness for last two months. Review of the hospital case records and patient history revealed that she had presented to the hospital two years back when she had been diagnosed as a case of dermatomyositis and put on oral corticosteroids. The patient's symptoms had subsided but she developed features of steroid psychosis. She left therapy and had been symptom free for around six months when the symptoms relapsed.

During current admission, her vitals were stable; general survey revealed no pallor or lymphadenopathy and only positive findings were the aforementioned skin changes. Respiratory and cardiovascular systems were normal on examination and abdomen was soft, non-tender with no evidence 
of organomegaly. Breast examination was normal. Neurological examination revealed findings limited to the motor system. Proximal muscles of both the shoulder and hip girdles had diminished power. Distal limb muscles were spared and so were the axial muscles. Sensory functions were intact and reflexes were preserved.

Our differentials for the skin lesions included cutaneous vasculitis, calciphylaxis and bacterial skin infection. Routine investigations returned a normal hematology, renal and hepatic panel. The serum creatine phosphokinase $(900$ $\mathrm{U} / \mathrm{L}$ ) and C-reactive protein titer were elevated. Electromyography revealed myopathic potentials (short duration, low-amplitude, polyphasic) and increased spontaneous activity. ANA, anti dsDNA, $c$ and $p$ ANCA and anti-Jo antibodies were all negative. CT scan of the thorax and ultrasonographic examination of the abdomen and pelvis did not locate an associated malignancy. Muscle biopsy revealed perivascular inflammatory cell infiltrate, preserved fascicular architecture, several foci of myophagocytosis, regenerative fibres, and perifascicular atrophy confirming our diagnosis of dermatomyositis. In the skin biopsy, we observed thickening of the epidermis, a perivascular inflammatory infiltrate comprising neutrophils and lymphocytes, along with few foci of inflammatory cells infiltrating the vessel walls and few foci of necrosis.

Our final diagnosis was cutaneous leukocytoclastic vasculitis in a patient of dermatomyositis. We started the patient on steroids and $10 \mathrm{mg}$ oral methotrexate weekly as a steroid sparing agent. Cyclophosphamide was not preferred in view of the reproductive age of the patient. The patient started responding to therapy by the $4^{\text {th }}$ week. The patient has been on regular follow-up for the last two years and stays free from significant muscle weakness or evidence of under lying malignancy. Her skin lesions have healed completely albeit with scarring [Fig.3]. We plan to screen her for other connective tissue

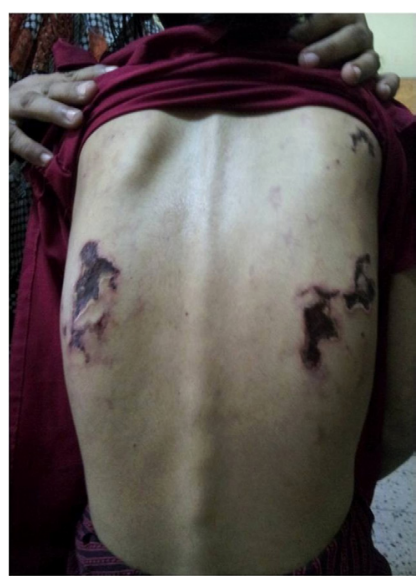

Fig.1: Ulcers on back of young girl with dermatomyositis.

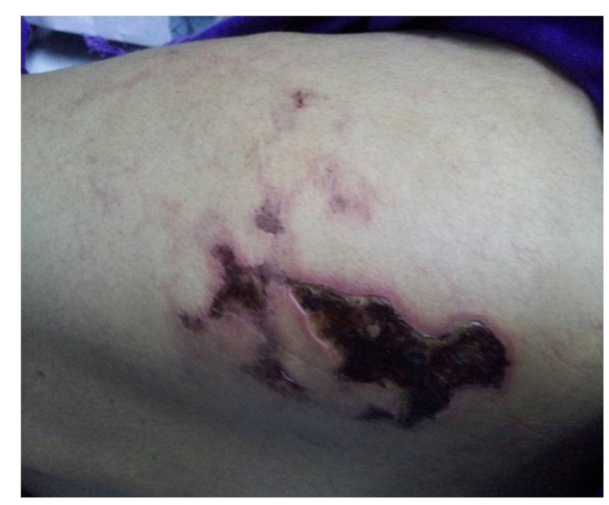

Fig.2: Ulcers on back (in close-up).

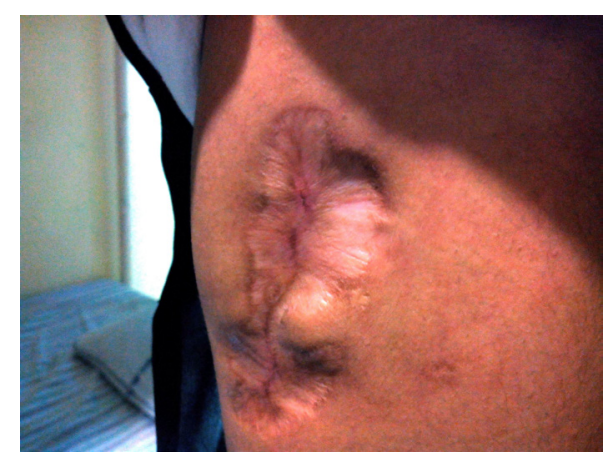

Fig.3: Ulcers resolved with scarring following treatment.

disorder or neoplasms at 3 monthly followups with general examination focused on lymphadenopathy, hepatosplenomegaly, breast and gynecological examination and chest radiography and ultrasonography of the abdomen for at least the next five years and at longer intervals thereafter. 


\section{Discussion}

A case series reports $9.2 \%$ of dermatomyositis/ polymyositis patients as having evidence of cutaneous vasculitis [3]. Alexander et al. in an Indian study report two out of 50 cases of cutaneous vasculitis to have underlying dermatomyositis [4]. Gupta et al. describe collagen profile positivity without overt manifestations in $10 \%$ of cutaneous vasculitis patients. It is evident that underlying connective tissue diseases let alone dermatomyositis are a rarity in cutaneous vasculitis cases [5]. The presence of cutaneous vasculitis in dermatomyositis patients has been considered as a predictor of underlying malignancy as well as a poor prognostic marker [6,7]. Alternatively vasculopathy has been suggested as a pathogenetic mechanism, responsible for the other cutaneous manifestations of dermatomyositis. Cutaneous vasculitis and occlusive vasculopathy are however more common in juvenile onset dermatomyositis. Kawakami et al. have described a case of cutaneous vasculitis in an adult patient with dermatomyositis and interstitial lung disease [8]. Case reports have also described urticarial vasculitis [9] and perforating non-vasculitic skin ulcers [10] in patients of dermatomyositis.

Therapy of dermatomyositis consists of steroids complemented by steroid sparing agents such as methotrexate, azathioprine and mycophenolate mofetil. Cyclophosphamide and dapsone have also been shown to have a crucial role in the management of refractory cutaneous complications of dermatomyositis including leukocytoclastic vasculitis.

\section{Conclusion}

Although cutaneous leukocytoclastic vasculitis has been described in the past in patients of dermatomyositis, extensive lesions such as in our patient especially without the backdrop of malignancy or another connective tissue disorder are a rarity. Despite the extensive lesions, a good response can be expected from steroid therapy supplemented by steroid sparing agents, as was observed in our patient.

Contributors: SSC: manuscript writing; AB: photographs and literature review; ISB: manuscript editing and literature review. ISG will act as guarantor. All authors approved the final version of manuscript.

Funding: None; Competing interests: None stated.

\section{References}

1. Choy EH, Isenberg DA. Treatment of dermatomyositis and polymyositis. Rheumatology (Oxford). 2002;41:713.

2. Dalakas MC, Hohlfeld R. Polymyositis and dermatomyositis. Lancet. 2003;362(9388):971-982.

3. Feldman D, Hochberg MC, Zizic TM, Stevens MB. Cutaneous vasculitis in adult polymyositis/ dermatomyositis. J Rheumatol. 1983;10:85-89.

4. Alexander B, Rameshkumar K, Jayaseelan E. Cutaneous vasculitis - a dynamic process posing diagnostic challenge. J Assoc Physicians India. 2003;51:574-577.

5. Gupta S, Handa S, Kanwar AJ, Radotra BD, Minz RW. Cutaneous vasculitis: Clinico-pathological Correlation. Indian J Dermatol Venereol Leprol. 2009;75:356-362.

6. Hunger RE, Dürr C, Brand CU. Cutaneous leukocytoclastic vasculitis in dermatomyositis suggests malignancy. Dermatology. 2001;202:123-126.

7. Tosun A, Serdaroğlu G, Aslan MT, Polat M, Akalin T, Tekgul H, et al. Severe juvenile dermatomyositis: two patients complicated with extra musculocutaneous involvement. Rheumatol Int. 2006;26:1040-1043.

8. Kawakami T, Mizoguchi M, Saito R, Soma Y. Histopathological evidence of small-vessel vasculitis within the skin and lungs associated with interstitial pneumonia in an adult patient with dermatomyositis. Clin Exp Dermatol. 2008;33:415-417.

9. Wang CC, Chen MJ, Ho HC, Hong HS. Urticarial vasculitis and dermatomyositis in a patient with nasopharyngeal carcinoma. Cutis. 2003;72:399-402.

10. Tomb R, Stephan F. Perforating skin ulcers occurring in an adult with dermatomyositis. Ann Dermatol Venereol. 2002;129:1383-1385. 